\title{
NEW RESULTS ON THE STRUCTURE AND STELLAR CONTENT OF THE OUTER REGIONS OF THE SMC
}

\author{
L.T. GARDINER \\ Department of Astronomy \\ University of Edinburgh \\ Edinburgh $\mathrm{EH} 9 \mathrm{BHJ}$ \\ United Kingdom \\ M.R.S. HAWKINS \\ Royal Observatory \\ Edinburgh $\mathrm{EH} 9 \mathrm{HHJ}$ \\ United Kingdon
}

\begin{abstract}
Surface distribution contour maps of the HB/clump population and the $<1$ Gyr main sequence population for the outer regions of the Small Magellanic Cloud (SMC) are presented. Aspects of the stellar population synthesis, large-scale structure and evolutionary history of the SMC halo are discussed.
\end{abstract}

Colour-magnitude data for 700,000 stars in ESO/SERC Fields 28, 29, 50, 51 and 52 have been obtained from COSMOS measurements of sets of $B$ and $R$ plates externally calibrated with CCD sequences obtained at the Danish $1.5 \mathrm{~m}$ telescope in Chile. The data reduction procedure for Fields 28 and 52 is described by Hatzidimitriou et al. (1989) and the associated study of the stellar population synthesis in the NE and SW outer SMC regions is presented by Hatzidimitriou (1989). The total studied area comprises approximately $80 \%$ of the outer area of the SMC beyond 2 degrees from the optical centre.

The red horizongal branch/clump (HB) population consists of stars of ages between 1 and 10 Gyr representing the intermediate-old stellar population of the SMC, whereas the main sequence (MS) population above $R=20.0$ (just above the plate limit at $R \simeq 20.2$ ) consists of stars of ages less than $1 \mathrm{Gyr}$ from consideration of the Yale isochrones. Magnitude and colour limits were used to define the HB and MS populations (Fig. 1). The number densities for each population were obtained for squares of size 5.6' $\times$ 5.6' and then smoothed by convolving with a Gaussian of $\sigma=8$. The surface contour maps for the HB/clump and main sequence populations are shown in Figs. 2 and 3.

The following results were obtained:

1. There is a marked difference in the projected distribution of the MS and HB populations with the MS deficient in the NW compared to the HB.

2. The isodensity contours for both populations are distorted towards the $\mathrm{E}$ and $\mathrm{SE}$ in a direction lying roughly towards the LMC. This is compatible with a scenario in which a recent ( $200 \mathrm{Myr}$ ago - Murai \& Fujimoto 1986) close passage of the LMC drew out stellar and gaseous material from near the central regions resulting in the formation of a stellar structure which is presently separating from the SMC (the Mini-Magellanic Cloud of Matthewson \& Ford, 1984).

3. The MS population can be separated into (a) an elliptical structure lying along the SMC major axis and (b) a tidal extension towards the LMC.

(c) 1991 IAU. Printed in the Netherlands. 


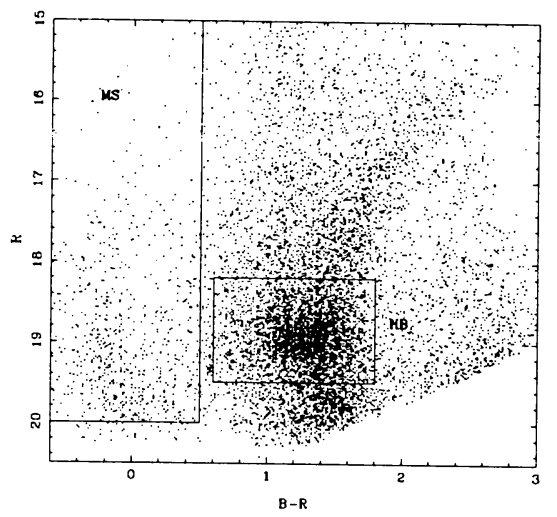

Figure 1. A typical example of a colour-magnitude diagram showing the MS and HB populations. The $\mathrm{CMMD}$ is for a $1 \mathrm{deg}^{2}$ region $2.5^{\circ} \mathrm{N}$ of the SMC optical centre.

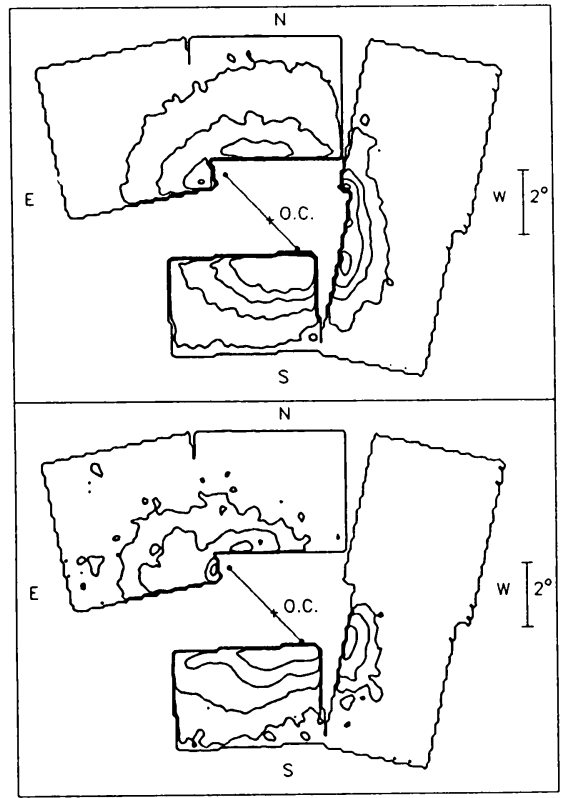

Figure 2. Contour map of the HB no. density. The number densities of the contours, increasing towards the optical centre, are 1700 , $3400,5200,6900$ stars per $\mathrm{deg}^{2}$.

The mosaics each consist of 5 UK Schmidt fields. The position of the optical centre $\left(R A=00^{\mathrm{h}} 51^{\mathrm{m}}, D E C=-73^{\circ}\right.$, de Vaucouleurs \& Freeman 1972) is shown, together with the semi-major axis of the SMC.

Figure 3. Contour map of the MS no. density. The number densities of the contours, increasing towards the optical centre, are 600 , $1100,2300,5700$ stars per $\mathrm{deg}^{2}$.

4. The halo defined by the HB extends to smaller radial distances from the SMC centre in the W and SW than in other directions indicating that these areas have been comparatively less affected by dynamical interactions with the LMC and Galaxy.

\section{References}

De Vaucouleurs, G., Freeman, K.C. (1972), Vistas Astr. 14, 163.

Hatzidimitriou, D. (1989), Ph.D thesis.

Hatzidimitriou, D., Hawkins, M.R.S., Gyldenkerne, K. (1989), MNRAS 241, 645.

Mathewson, D.S., Ford, V.L. (1984), IAU Symp. 108 Structure and Evolution of the Magellanic Clouds, S. van den Bergh and K.S. de Boer (eds.) (Reidel: Dordrecht) p.125.

Murai, T., Fujimoto, M. (1986), Astrophys. Sp. Sci. 119, 169. 